\title{
Treatment and prognosis of primary malignant melanoma of the esophagus
}

\author{
Lei Cheng ${ }^{1,2}$, Zheng-Ying Guo ${ }^{2}$, Lei Lei ${ }^{3}$, Wen-Xian Wang ${ }^{3}$, Chun-Wei Xu ${ }^{4}$, Mei-Yu Fang ${ }^{3}$ \\ ${ }^{1}$ Department of Thoracic Radiotherapy, Chinese Academy of Sciences University Cancer Hospital (Zhejiang Cancer Hospital), Hangzhou, China; \\ ${ }^{2}$ Institute of Cancer and Basic Medicine (ICBM) of Chinese Academy of Sciences, Hangzhou, China; ${ }^{3}$ Department of Chemotherapy, Chinese \\ Academy of Sciences University Cancer Hospital (Zhejiang Cancer Hospital), Hangzhou, China; ${ }^{4}$ Department of Respiratory Medicine, Jinling \\ Hospital, Nanjing University School of Medicine, Nanjing, China \\ Contributions: (I) Conception and design: L Cheng, CW Xu, MY Fang; (II) Administrative support: None; (III) Provision of study materials or \\ patients: L Cheng, ZY Guo, L Lei, WX Wang; (IV) Collection and assembly of data: L Cheng, L Lei; (V) Data analysis and interpretation: L Cheng, \\ L Lei, WX Wang; (VI) Manuscript writing: All authors; (VII) Final approval of manuscript: All authors. \\ Correspondence to: Mei-Yu Fang, MD, PhD. Department of Chemotherapy, Chinese Academy of Sciences University Cancer Hospital (Zhejiang \\ Cancer Hospital), No. 1 Banshan East Road, Gongshu District, Hangzhou, China. Email: fangmy@zjcc.org.cn; Chun-Wei Xu, MD, PhD. \\ Department of Respiratory Medicine, Jinling Hospital, Nanjing University School of Medicine, 305 Zhongshan Road, Nanjing, China. \\ Email: xuchunweibbb@163.com.
}

Background: Primary malignant melanoma of the esophagus (PMME) is rare with high malignancy and poor prognosis. The aim of this study was to investigate the relationship between prognosis and clinicopathological characteristics of this disease.

Methods: A total of 9 patients with PMME were treated in Zhejiang Cancer Hospital between 2009 and 2019 retrospectively. According to 8th edition AJCC/UICC staging of cancers of the esophagus and esophagogastric junction, none of the patients were in stage I. However, 5 patients were in stage II, 2 patients were in stage III, and 2 patients were in stage IV at diagnosis. Five patients received surgery, while one of them received palliative resection. Three patients received postoperative chemotherapy; two of them $(2 / 5)$ were diagnosed with recurrence. One patient in stage II received targeted therapy. One patient in stage III received first line chemotherapy and efficacy evaluation was stable disease (SD). Another one in stage III received biotherapy. One patient in stage IV received Chinese Medicine treatment and another received chemotherapy and palliative surgery.

Results: The 1-year disease-free survival (DFS) and overall survival (OS) rates of stage II who received surgery were $50 \%(2 / 4)$ and $100 \%$ (4/4) respectively. The 2 -year DFS and OS rates were 50\% (2/4) and $75 \%$ (3/4), respectively. However, patients with stage III-IV have a very poor prognosis. The $1-y$ ear OS is $0 \%$.

Conclusions: Due to the small sample size, the statistic efficacy is low, but it can provide a certain theoretical basis for future research.

Keywords: Malignant melanoma; immunohistochemistry; human melanoma protein 45 (HMB-45); S-100; BRAF 600; programmed death-1 (PD-1)

Submitted Nov 01, 2019. Accepted for publication Feb 05, 2020.

doi: $10.21037 /$ tcr-19-2349

View this article at: http://dx.doi.org/10.21037/tcr-19-2349 


\section{Introduction}

Primary malignant melanoma of the esophagus is a rare disease which has been described only occasionally in case reports. It accounts for only $0.1 \%$ to $0.5 \%$ of all primary esophageal malignancies (1). Due to the improvement of equipment, and a higher awareness of the disease, in the general population, the early diagnosis rate of esophageal malignancies has improved. However, because the disease is easy to metastasize early, the survival rate is still low. This article lists the patients with esophageal malignant melanoma admitted to our hospital in the past ten years, as well as their related diagnosis, treatment and prognosis, in order to further understand the disease.

\section{Methods}

\section{Patients}

Patients with PMME who had undergone treatment at Zhejiang Cancer Hospital between January 2009 and September 2019 were retrospectively identified (Table 1). Patients' clinical stages were determined according to the 2002 American Joint Committee on Cancer (AJCC) tumor-node-metastasis (TNM) classification. Recurrence or metastases were confirmed using chest computed tomography (CT), as well as bone scan and/or computed tomography of the abdomen. The histologic types were determined based on the 2004 WHO classification. Pathological examination of surgically removed specimens. Immunohistochemical staining was performed using human melanoma protein 45 (HMB-45), melanin A protein (Melan-A), S-100 protein, and cytokeratin (CK) (Table 2). Patients who died from another disease not related to Malignant melanoma were excluded from the current study. The study was reviewed and approved by the Institutional Ethics Committee of Zhejiang Cancer Hospital (IRB-2020-44), and conducted in accordance with the Declaration of Helsinki (as revised in 2013). Written informed consent was obtained from all patients.

\section{Follow-up}

For patients who underwent a surgical intervention, all were examined in the outpatient clinic at 3-month intervals for the first 2 years and, thereafter, at 6 -month intervals. For patients at an advanced stage, the follow-ups were at 6 to 8 weeks apart. The last follow-up time point was September, 30, 2019. The median follow-up of patients was 48 months, ranging from 17 to 92 months.

\section{Statistical analysis}

Disease-free survival (DFS) covered the time from surgery to progression or death from any cause. Progression free survival (PFS) was defined as the date from the date of treatment to the confirmation of overall disease progression. The definition of overall survival (OS) was based on the date of treatment and the most recent follow-up or date of death. Assuming $\mathrm{P}<0.05$ is statistically significant, statistical analysis was performed using SPSS version 19 (SPSS Inc., Chicago, Illinois, USA). Evaluation of DFS, PFS and OS using Kaplan-Meier method and log-rank test.

\section{Results}

\section{Patient characteristics}

According to 2002 TNM classification, 9 patients of malignant melanoma were included in the study. There were 6 males and 6 females in this group, aged 46-74 years, with a median age of 62 years. Clinical characteristics of all patients are listed in Table 1. According to 2002 TNM classification, stage II in 5 patients, stage III in 2 patients, and IV in 2 patients at diagnosis. In case 9, computed tomography (CT) scans revealed huge tumor in the lower esophagus (Figure 1A) and multiple nodules in the abdominal cavity at diagnosis (Figure 1B). Esophagoscope revealed filling defect in the lower part of the esophagus (Figure 1C). Blue-violet nodule visible under gastroscope (Figure 1D).

All nine cases showed similar morphological characters, the presence of malignant melanoma. Needle biopsy showed malignant melanoma in (Figure 2A). Immunohistochemistry results showed HMB-45 positive in case 9 (Figure $2 B$ ). The patient was also positive in Melan-A, Vimentin, S-100 and negative in CK, EMA, LCA, Syn, CD99.

\section{Treatment and prognosis}

Four patients received surgery and three of them were received postoperative chemotherapy, two of them (2/4) were diagnosed with recurrence. For stage II patient (case 5), he received postoperative radiotherapy and chemotherapy with two cycles of TP regimen (docetaxel $75 \mathrm{mg} / \mathrm{m}^{2}$ $\mathrm{d} 1$; Cisplatin $75 \mathrm{mg} / \mathrm{m}^{2} \mathrm{~d} 1$ ). Disease progression was in October 2018 and the DFS was 10.0 months. The patient showed the presence of metastases in Mediastinal lymph node. Needle biopsy indicated the pathological pattern was Malignant melanoma. Then he received chemotherapy in 
Table 1 Characteristics of 9 patients in malignant melanoma

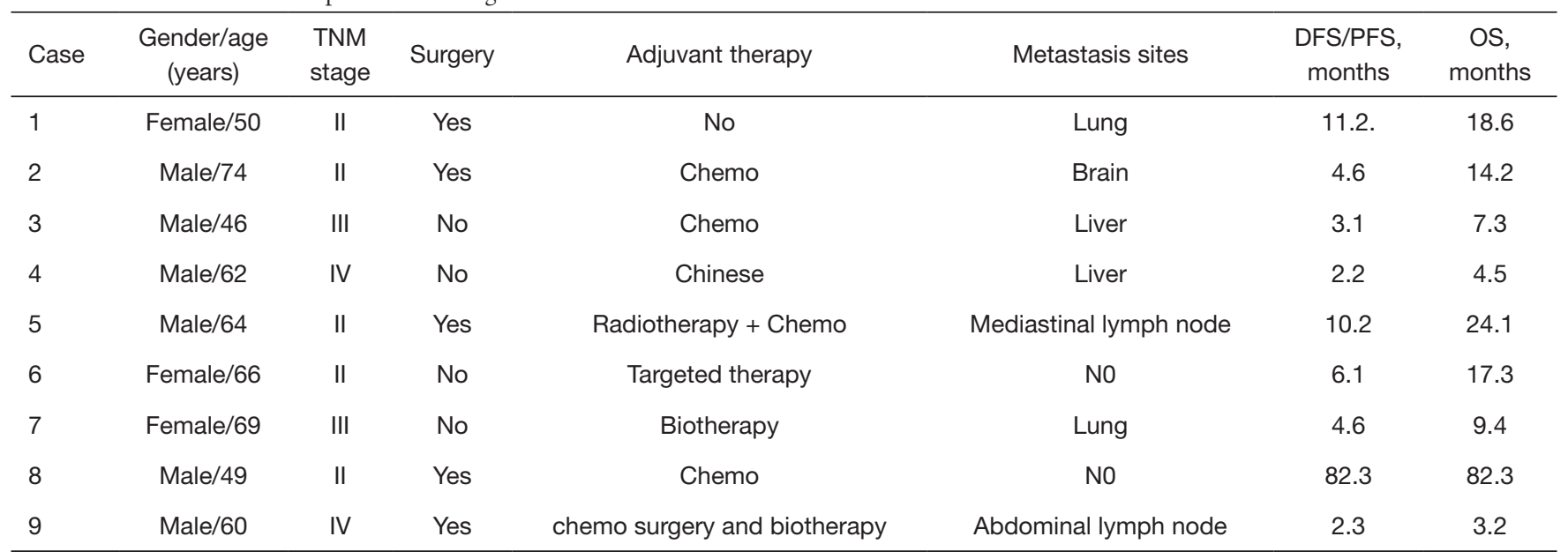

Table 2 Hematoxylin and eosin staining and immunohistochemistry results of 9 patients with PMME

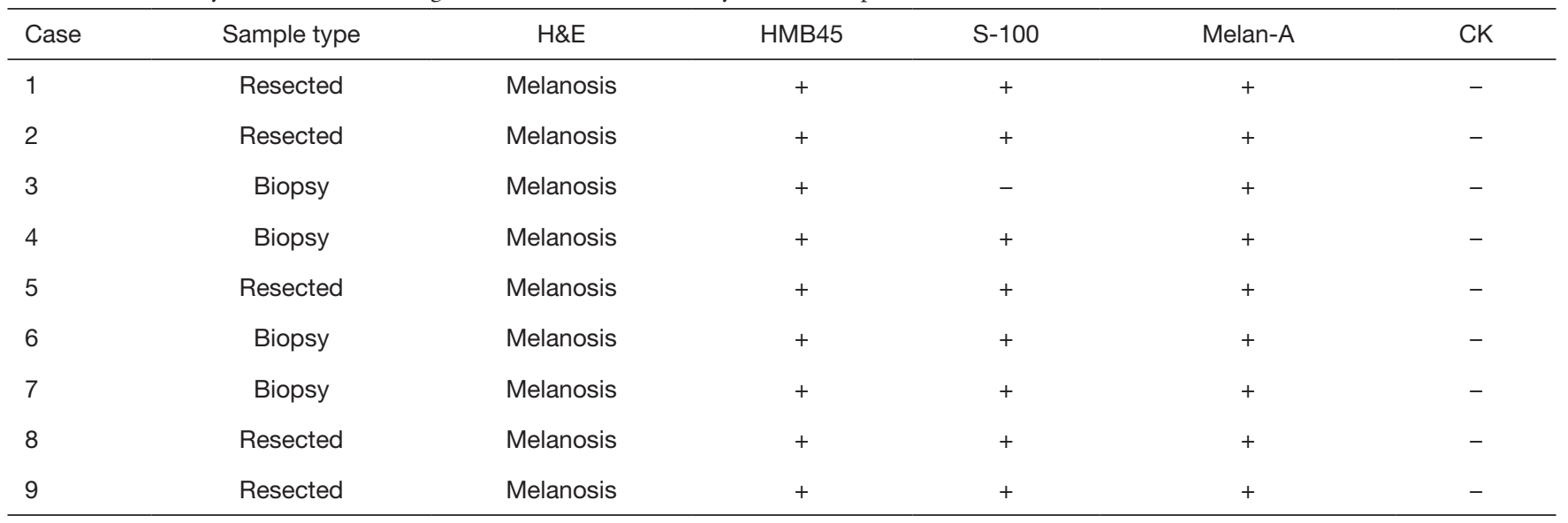

H\&E, hematoxylin and eosin staining; HMB45, human melanin black 45; CK, cytokeratin; +, positive; -, negative.

January 2019 with two cycles of TMZ (temozolomide) + cisplatin+ anti-angiogenesis treatment and supraclavicular and mediastinal lymph node tumor radiotherapy. The patient was alive with no evidence of progression disease to date.

For Stage III patient (case 3), he received chemotherapy with two cycles (Temozolomide $150 \mathrm{mg} / \mathrm{m}^{2}+$ Cisplatin 25 $\mathrm{mg} / \mathrm{m}^{2}+\mathrm{rh}$-Endostin $\left.15 \mathrm{mg} / \mathrm{d} \mathrm{d} 1-14\right)$. However, after 2 cycles of chemotherapy, efficacy evaluation was progressive disease (PD). The patient eventually died of liver metastasis.

For stage IV patient (case 9), he received chemotherapy (Oxaliplatin $130 \mathrm{mg} / \mathrm{m}^{2}+$ Fluorouracil $500 \mathrm{mg} / \mathrm{m}^{2}+$ docetaxel $75 \mathrm{mg} / \mathrm{m}^{2}$ ) in December 2008 with two cycles with and efficacy evaluation was stable disease (SD). However, the patient experienced gastrointestinal bleeding in February 2009. Thoracic surgeons believed that although the patient was staged late, there was no indication for surgery, but the patient had bleeding, palliative surgery to remove bleeding lesions, which may have been helpful to his life. The esophageal tumor biopsy showed the malignant melanoma. Unfortunately, the patient eventually died of lung infection 20 days later.

The 1-year DFS and OS were 50\% (2/4) and 100\% (4/4), respectively. The 2-year DFS and OS were 50\% (2/4) and $75 \%(3 / 4)$, respectively.

\section{Discussion}

The incidence of melanoma in China is low, but it has shown a rapid growth in recent years, with an annual 

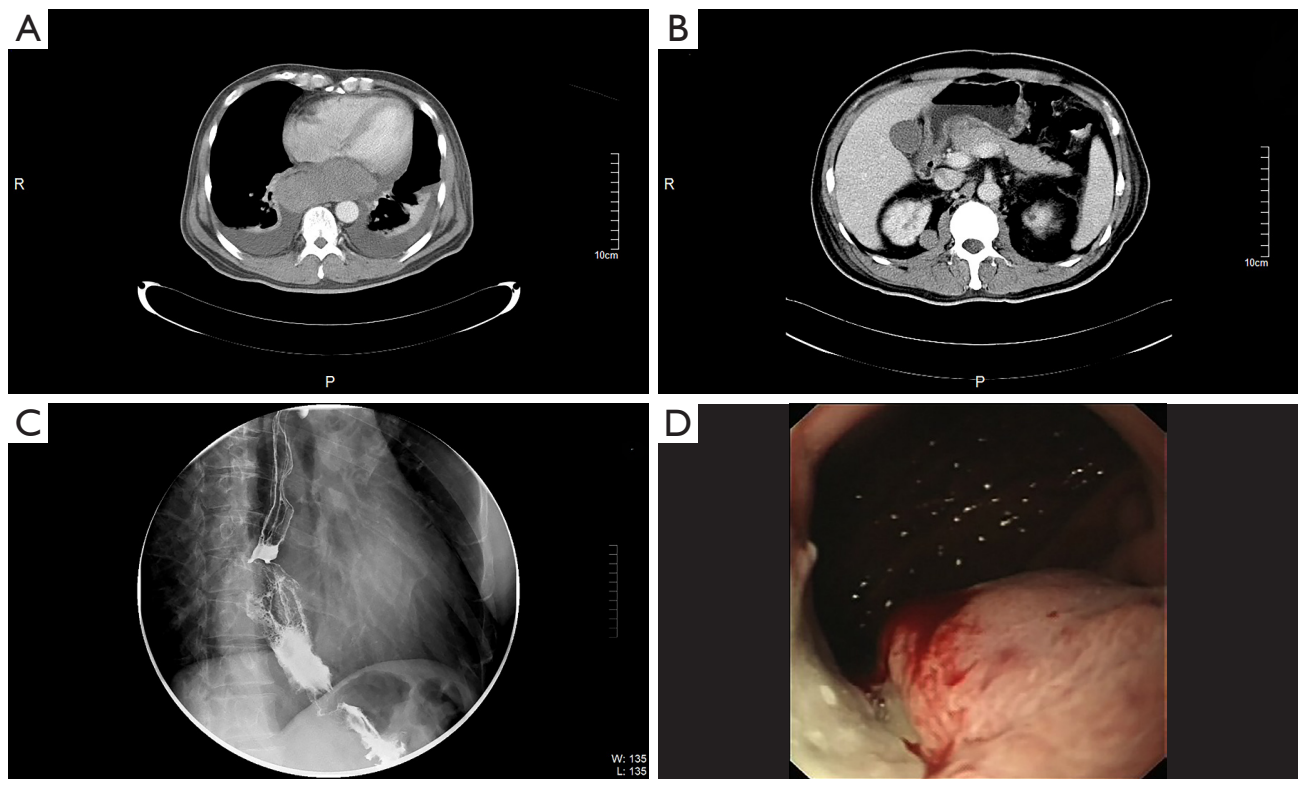

Figure 1 CT scans in case 9. (A) CT scans showed a large tumor in the lower part of the esophagus. (B) Multiple nodules in the abdominal cavity at diagnosis. (C) Esophageal angiography showed filling defect in the lower part of the esophagus. (D) Blue-violet nodule visible under gastroscope.
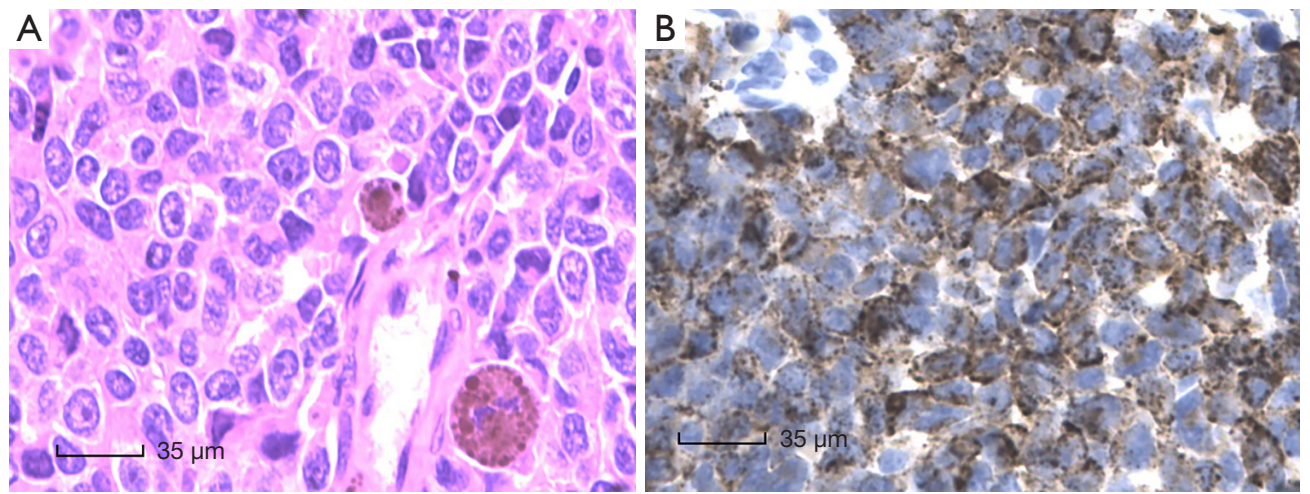

Figure 2 Pathology of HE in malignant melanoma. (A) Needle biopsy showed malignant melanoma in case 9 (HE, $\times 400$ ). (B) Immunohistochemistry results showed HMB-45 positive in case $9(\mathrm{HE}, \times 400)$.

incidence of about 20,000 new cases. Melanoma can occur in various parts of the body, most commonly on the surface of the skin, followed by the uvea, head and neck, digestive tract and genitourinary mucosa. Malignant melanin originating in the digestive tract. The tumor is mostly rectal anal canal, accounting for all malignant melanoma $0.5-2.0 \%$.

Esophageal malignant melanoma often occurs in the lower and middle esophagus, which is reported to account for about $90 \%$, and the incidence of men is significantly higher than that of women (2). The incidence of the middle and lower position is similar to the data of our team $(88.9 \%)$. Studies have shown that the median age of diagnosis is 60.5 years, and the ratio of male to female is 2:1 (3). The clinical manifestations of the disease are similar to those of other esophageal malignancies. Patients often experience eating sensation, non-specific post-sternal pain, acid reflux and other gastrointestinal symptoms and weight loss, occasionally hematemesis and melena, and some patients may be asymptomatic. Currently, most PMME are 
reported in the case, and there are few large retrospective or prospective studies. The early diagnosis and treatment of esophageal malignant melanoma is still difficult due to the lack of support from large clinical data. PMME has a poor prognosis with a 5 -year survival rate of less than $5 \%$ (4). A retrospective clinical study at Peking University included 76 patients with an OS of only 22.3 months (95\% CI: 16.4-28.2). Stage IV and stage III patients were 19.5 months. The OS for one year and two years was $79.2 \%$ and $38.9 \%$ respectively. The median OS of patients who were newly diagnosed as distant patients was 15.8 months, and the average survival rate of patients with stage III disease with metastatic or unrespectable resection was 22.8 months from the date of first diagnosis $(\mathrm{P}=0.032)(5)$.

At present, endoscopic biopsy is still one of the effective methods for diagnosing malignant melanoma. Primary esophageal melanoma is often covered by squamous epithelium, and melanocytes tend to concentrate on the lesion, which makes it difficult to identify lesions under endoscopy (6). Therefore, Pathologist are always using immunohistochemistry (IHC) to confirm the diagnosis. In the review of the literature, the rate of positivity with cytokeratin was reported to be $7 \%$ (7). In most cases, HMB-45, Melan-A, and S-100 are simultaneously expressed instead of express CK. S-100 seems to be the most sensitive biomarker for melanoma, while HMB-45 and Melan-A demonstrate relatively good specificity but not as good sensitivity as S-100 (8). HMB-45 is an anti-melanomaspecific monoclonal antibody that is $100 \%$ specific for malignant melanoma. The tumor cells in our group were diffusely expressed HMB-45, Melan-A, S-100 scattered, CK negative, consistent with the literature reports (9). In our cases, the rate of HMB-45 is $100 \%$, and so does S-100 and Melan-A.

Due to the rare disease, there is no international consensus on the treatment of esophageal melanoma. But surgery is still a common treatment for PMME. In our group, five patients received surgery while one of them was palliative resection. However, it has been reported in the literature that the interval between the initial surgery and the recurrence is only 4.5 months. Therefore, there are more experts to explore postoperative adjuvant therapy treatment. Also, three of them received postoperative chemotherapy in our group. As early as 2013, researchers have suggested that TMZ-based adjuvant chemotherapy can improve Recurrence-free survival (RFS) and OS of mucosal melanoma (10). However, Chemotherapy is not satisfactory for patients with advanced PMME. Weiner et al. reported OS of chemotherapy of only 7.7 months and 3-year OS of 0 in eight patients (11). Wang et al. (5) reported the overall response rate (ORR) of chemotherapy was only $10.9 \%$ with a short PFS of three months also. In the past five years, with the development of targeted therapies and immunotherapy, there are more options for the treatment of advanced melanoma. Due to the large span of the age, and the lack of understanding of mucosal malignant, a large number of mucosal patients have not been genetically tested; in domestic research, the kit mutation is about $20 \%$. BRAF $60016 \%$ or so mutation. Up to $25 \%$ of patients with cutaneous melanoma have BRAF 600 mutations. The BRAF inhibitors vemurafenib and dabrafenib improve survival in advanced patients. However, compared to the skin, mucosal malignant BRAF mutation rates are lower.

Mucosal melanoma is one of the major subtypes of melanoma in China. It is not sensitive to traditional chemotherapy, and the response rate to single-agent immunotherapy is extremely limited. Immunotherapy may be a new way out. In recent years, studies have shown that anti-PD-1 (programmed death-1) antibodies have achieved a certain effect on patients with advanced melanoma. Some of data is derived from the treatment of mucosal melanoma. D'Angelo et al. (12) reported PD-1 checkpoint inhibitor monotherapy achieves unsatisfactory clinical outcomes in patients with mucosal melanoma for median progressionfree survival was 3.0 months ( $95 \%$ CI, 2.2 to 5.4 months), for patients who were treated with nivolumab combined with ipilimumab, Median progression-free survival was 5.9 months. Meanwhile, the incidence of grade 3 or 4 treatment-related adverse events also increased significantly.

Wang et al. (5) reported PD-1checkpoint inhibitors proved to be activity against PMME. Results suggested a response rate of $75 \%$ with 11.4 months median duration of response. Additionally, multivariate survival analysis showed that IT systemic treatment was correlated with a significant OS benefit. Some of researchers shows that monotherapy with PD-1 checkpoint inhibitor alone is not effective in treating mucosal melanoma, especially in patients with visceral metastases (12), the ORR rate is only $17.65 \%$ (13). However, other studies have shown that the response rate in esophageal malignant melanoma is higher. At present, the Beijing Cancer Hospital of China has published a conclusion that with the axitinib plus toripalimab reach to $70 \%$. The disease control rate (DCR) of axitinib combined with toripalimab is as high as $86 \%$, the ORR was $51.7 \%$, and the mean response time (TTR) was 2.1 months. The median PFS was 7.5/8.9 months and the median OS had 
not been reached. The mucosal malignant monotherapy PD1 has low efficacy, but combining this with antivascular multi-target inhibitors significantly improve the efficacy (14). The mucosal malignant monotherapy PD1 has low efficacy, but combined with anti-vascular multi-target inhibitors significantly improve the efficacy. Therefore, more basic research is needed, including mechanisms, biomarkers, etc. for further validation.

The major limitations of our study are its retrospective analysis and small number of patients. However, with no cases in prospective clinical studies and treatment is lack, our retrospective study can also be considered to be meaningful.

\section{Conclusions}

In conclusion, PMME is a rare, with the discovery of BRAF or KIT gene mutations, immunotherapy offers new methods in the treatment of patients, but complete surgical resection remains the primary treatment. I hope that more randomized controlled trials will lead to reliable data to develop primary malignant melanoma of the esophagus diagnosis and treatment specifications.

\section{Acknowledgments}

Funding: This study was supported in part by grants from the Medical Scientific Research Foundation of Zhejiang Province of China (2019RC027) and Xisike-Hanson Cancer Research Foundation (Y-HS2019-20).

\section{Footnote}

Conflicts of Interest: All authors have completed the ICMJE uniform disclosure form (available at http://dx.doi. org/10.21037/tcr-19-2349). The authors have no conflicts of interest to declare.

Ethical Statement: The authors are accountable for all aspects of the work in ensuring that questions related to the accuracy or integrity of any part of the work are appropriately investigated and resolved. The study was reviewed and approved by the Institutional Ethics Committee of Zhejiang Cancer Hospital (IRB-2020-44), and conducted in accordance with the Declaration of Helsinki (as revised in 2013). Written informed consent was obtained from all patients for publication of this manuscript and any accompanying images.
Open Access Statement: This is an Open Access article distributed in accordance with the Creative Commons Attribution-NonCommercial-NoDerivs 4.0 International License (CC BY-NC-ND 4.0), which permits the noncommercial replication and distribution of the article with the strict proviso that no changes or edits are made and the original work is properly cited (including links to both the formal publication through the relevant DOI and the license). See: https://creativecommons.org/licenses/by-nc-nd/4.0/.

\section{References}

1. Volpin E, Sauvanet A, Couvelard A, et al. Primary malignant melanoma of the esophagus: a case report and review of the literature. Dis Esophagus 2002;15:244-9.

2. Cardeña R, Yep-Gamarra V, Donet-Mostacero J, et al. Primary melanoma of the esophagus. Rev Gastroenterol Peru 2012;32:303-8.

3. Iwanuma Y, Tomita N, Amano T, et al. Current status of primary malignant melanoma of the esophagus: clinical features, pathology, management and prognosis. J Gastroenterol 2012;47:21-8.

4. Kido T, Morishima H, Nakahara M, et al. Early stage primary malignant melanoma of the esophagus. Gastrointest Endosc 2000;51:90-1.

5. Wang X, Kong Y, Chi Z, et al. Primary malignant melanoma of the esophagus: A retrospective analysis of clinical features, management, and survival of 76 patients. Thorac Cancer 2019;10:950-6.

6. Sabanathan S, Eng J, Pradhan GN. Primary malignant melanoma of the esophagus. Am J Gastroenterol 1989;84:1475-81.

7. Micke O, Schäfer U, Glashörster M, et al. Radiationinduced esophageal carcinoma 30 years after mediastinal irradiation: case report and review of the literature. Jpn J Clin Oncol 1999;29:164-70.

8. Imai S, Suzuki A, Yamamoto Y, et al. Primary malignant melanoma of esophagus following chemoradiotherapy for esophageal squamous cell carcinoma: report of a case. Clin J Gastroenterol 2017;10:336-41.

9. Navarro-Ballester A, De Lazaro-De Molina S, GaonaMorales J, et al. Primary Malignant Melanoma of the Esophagus: A Case Report and Review of the Literature. Am J Case Rep 2015;16:491-5.

10. Lian B, Si L, Cui C, et al. Phase II randomized trial comparing high-dose IFN- $\alpha 2 \mathrm{~b}$ with temozolomide plus cisplatin as systemic adjuvant therapy for resected mucosal melanoma. Clin Cancer Res 2013;19:4488-98. 
11. Weiner JP, Shao M, Schwartz D, et al. Patterns of care and survival outcomes in the treatment of esophageal melanoma. Dis Esophagus 2017;30:1-6.

12. D'Angelo SP, Larkin J, Sosman JA, et al. Efficacy and Safety of Nivolumab Alone or in Combination With Ipilimumab in Patients With Mucosal Melanoma: A Pooled Analysis. J Clin Oncol 2017;35:226-35.

13. Maeda T, Yoshino K, Nagai K, et al. Efficacy of nivolumab monotherapy against acral lentiginous melanoma and

Cite this article as: Cheng L, Guo ZY, Lei L, Wang WX, Xu CW, Fang MY. Treatment and prognosis of primary malignant melanoma of the esophagus. Transl Cancer Res 2020;9(7):41414147. doi: $10.21037 /$ tcr-19-2349 mucosal melanoma in Asian patients. Br J Dermatol 2019;180:1230-1.

14. Sheng X, Yan X, Chi Z, et al. Axitinib in Combination With Toripalimab, a Humanized Immunoglobulin G4 Monoclonal Antibody Against Programmed Cell Death-1, in Patients With Metastatic Mucosal Melanoma: An Open-Label Phase IB Trial. J Clin Oncol 2019;37:2987-99. 